https://doi.org/10.23913/rics.v8i16.80

Artículos Científicos

\title{
Nutrición enteral temprana con inmunonutrientes en pacientes con traumatismo craneoencefálico en la unidad de cuidados intensivos
}

Early Enteral Nutrition with Immunonutrients in Patients with Traumatic Brain Injury in the Intensive Care Unit

Nutrição enteral precoce com imunonutrientes em pacientes com trauma cranioencefálico em unidade de terapia intensiva

Gisela Ponce y Ponce de León

Universidad Autónoma de Baja California, Baja California, México giselaponce@uabc.edu.mx https://orcid.org/0000-0001-9708-3102

José de Jesús Mayagoitia Witrón Universidad Autónoma de Baja California, Baja California, México josemaya01@prodigy.net.mx https://orcid.org/0000-0002-7388-157X

José Manuel Cornejo Bravo Universidad Autónoma de Baja California, Baja California, México jmcornejo@uabc.edu.mx https://orcid.org/0000-0002-0013-8937

María Eugenia Pérez Morales Universidad Autónoma de Baja California, Baja California, México eugenia@uabc.edu.mx https://orcid.org/0000-0001-5810-0694

\section{(c) $9 \Leftrightarrow$}

Esta obra está bajo licencia internacional Creative Commons

Reconocimiento-Nocomercial- 
Ulises Rieke Campoy

Universidad Autónoma de Baja California, Baja California, México

rieke@uabc.edu.mx

https://orcid.org/0000-001-5087-7933

Alejandro Mayagoitia Ponce

Universidad Autónoma de Guadalajara. Escuela de Medicina, Jalisco, México alejandromayagoitia026@gmail.com https://orcid.org/0000-0001-9515-1618

\section{Resumen}

El traumatismo craneoencefálico (TCE) es la tercera causa de muerte en México dentro de la categoría de muertes violentas y accidentes, por lo que se considera un grave problema de salud pública. Se ha observado un beneficio de la inmunonutrición temprana en pacientes con TCE. El objetivo de este trabajo fue determinar el impacto de la nutrición enteral temprana con inmunonutrientes (NETI) en la morbimortalidad de pacientes con TCE dentro de la Unidad de Cuidados Intensivos (UCI) del Hospital General de Mexicali. Se trata de un ensayo clínico aleatorizado en el que se incluyeron todos los pacientes con TCE que ingresaron a la UCI del 1 de agosto de 2016 al 30 de junio de 2017. Se conformaron dos grupos: el grupo de estudio sometido a NETI y un grupo control que fue sometido a nutrición enteral temprana licuada (NETL).

Del total de pacientes, 16 fueron hombres $(72.7 \%)$ y 6 mujeres $(27.3 \%)$, con una media de edad de 34.7 años. Las principales causas del TCE fueron por atropellamiento y accidente de tránsito (54.6 \%). El grupo con NETI, en relación con el grupo control, presentó aumento de peso ( $\mathrm{t}$ de Student de 23.73; $p=0.001$ ), de proteínas totales ( $\mathrm{t}$ de Student de 13.40; $p=0.001$ ), de leucocitos (t de Student de 2.05; $p=0.002$ ) y linfocitos al egreso de la UCI (t de Student de 7.09; $p=0.001$ ), con disminución en casos de neumonía (t de Student de 13.78; $p=0.001$ ), infección de vías urinarias (t de Student de 8.07; $p=0.001$ ), días de estancia en la UCI ( $\mathrm{X}^{2}$ Spearman -.432; $\left.p=0.05\right)$, días sometidos a ventilación mecánica $\left(\mathrm{X}^{2}\right.$

\section{(c) $9 \Leftrightarrow$}


Spearman -.432; $p=0.05)$ y de nutrición enteral $\left(\mathrm{X}^{2}\right.$ Spearman $\left.-.425 ; p=0.05\right)$. La mortalidad fue menor en el grupo de NETI sin reportarse una diferencia significativa.

Los pacientes con TCE que recibieron NETI presentaron una disminución en la incidencia de complicaciones infecciosas, así como respecto al tiempo de estancia intrahospitalaria, gracias a lo cual es posible mejorar en menos tiempo la salud del paciente y a la vez reducir costos debidos a la hospitalización.

Palabras clave: nutrición enteral temprana, nutrición enteral con inmunonutrientes, traumatismo craneoencefálico, unidad de cuidados intensivos.

\section{Abstract}

Early enteral nutrition is initiated in the first $24-48$ hours after injury or admission to the intensive care unit (ICU). It is a nutritional support method with beneficial effects that improves the patient's outcome. Traumatic brain injury (TBI) is the third cause of death in México related to accidents and violence; therefore, it is considered a serious public health problem.

The objective of this study was to determine if early enteral nutrition with immunonutrients (EENI) supplied to TBI patients diminishes morbidity and mortality in Mexicali`s General Hospital ICU.

It was a randomized clinical study. Patients that were included were those admitted to the ICU during the time period from August $1^{\text {st }}$ of 2016 to June $30^{\text {th }}$ of 2017 that fulfilled the inclusion criteria. Two groups were formed: one was the control group submitted to early enteral nutrition with blended diet and the other group of study which was submitted to EENI.

The study included 16 males (72.7\%) patients and 6 females (27.3\%), with an average age of 34.7 years. The main causes of TBI were run over and motor vehicle accidents in 54.6\%. The group with EENI, when compared to the control group, showed an increase in weight (Student T 23.737, p=.001), total proteins (Student T 13.40, p=.001), white blood cells (Student $\mathrm{T}$ of 2.05, p=.002) and lymphocytes (Student $\mathrm{T}$ of 7.09, $\mathrm{p}=.001$ ) at their

\section{(c) 9 (F)}

Esta obra está bajo licencia internacional Creative Commons

Reconocimiento-Nocomercial- 
discharge from the ICU, with decrease in pneumonia (t de Student 13.78, p=0.001), urinary tract infection (t de Student 8.07, p=0.001) and the length of stay at the ICU (X $\mathrm{X}^{2}$ Spearman $.432, \mathrm{p}=0.05)$, the days of mechanical ventilation $\left(\mathrm{X}^{2}\right.$ Spearman $\left.-.432, \mathrm{p}=0.05\right)$ and enteral nutrition $\left(\mathrm{X}^{2}\right.$ Spearman -.425, $\left.\mathrm{p}=0.05\right)$. The mortality was less in the EENI group without any significant difference reported.

EENI can have a positive impact, decreasing the incidence of infectious complications and length of stay in the ICU, therefore improving patient's health in less time and also reducing hospitalization costs.

Keywords: early enteral nutrition, enteral nutrition with immunonutrients, traumatic brain injury, intensive care unit.

\section{Resumo}

Lesão cerebral traumática (TCE) é a terceira causa de morte no México dentro da categoria de mortes e acidentes violentos, razão pela qual é considerado um grave problema de saúde pública. Um benefício da imunonutrição precoce foi observado em pacientes com TCE. O objetivo deste estudo foi determinar o impacto da imunonutrientes primeiros Nutrição Enteral (NETI) sobre a morbidade e mortalidade de pacientes com TCE na Unidade de Terapia Intensiva (UTI) do Hospital Geral de Mexicali. Este é um ensaio clínico randomizado em que todos os pacientes com TCE internados em UTI 01 de agosto de 2016 a 30 de Junho de 2017. Foram estudados dois grupos foram incluídos: o grupo de estudo sob Neti e um grupo controle que foi submetido a nutrição enteral precoce liquefeita (NETL).

Do total de pacientes, 16 eram homens $(72,7 \%)$ e 6 mulheres $(27,3 \%)$, com idade média de 34,7 anos. As principais causas do TCE foram atropelamento e acidente de trânsito $(54,6 \%)$. O grupo neti, em relação ao grupo de controlo mostrou aumento de peso (t de Student 23,73; $\mathrm{p}=0,001$ ), total (t de Student 13,40; $\mathrm{p}=0,001$ ) de proteína, de leucócitos (t de Student 2,05; $\mathrm{p}=0,002$ ) e linfócitos para descarregar da UTI ( $\mathrm{t}$ de Student 7,09; $\mathrm{p}=0,001$ ), com diminuição dos casos de pneumonia ( $\mathrm{t}$ de Student 13,78; $\mathrm{p}=0,001$ ), infecção do trato urinário ( $\mathrm{t}$ estudante 8,07; $\mathrm{p}=0,001$ ) dias, o UTI (X2 Spearman -.432; $\mathrm{p}=0,05)$, dias sob

\section{(c)}

Esta obra está bajo licencia internacional Creative commons Reconocimiento-Nocomercial- 
Revista Iberoamericana de las Ciencias de la Salud

ventilação mecânica (X2 Spearman -.432; p =0,05) e nutrição entérica (X2 Spearman -425, $\mathrm{p}=0,05)$. A mortalidade foi menor no grupo NETI sem relatar uma diferença significativa. pacientes com TCE que receberam NETI mostraram uma diminuição na incidência de complicações infecciosas e com o tempo de permanência hospitalar, graças ao qual é possível melhorar em menos tempo a saúde do paciente e, ao mesmo tempo, reduzir os custos devido à hospitalização.

Palavras-chave: nutrição enteral precoce, nutrição enteral com imunonutrientes, traumatismo cranioencefálico, unidade de terapia intensiva.

Fecha Recepción: Diciembre 2018

Fecha Aceptación: Mayo 2019

\section{Introducción}

El soporte nutricional ha pasado a ser un componente esencial en el manejo del paciente en estado crítico (Torres et al., 2008; García y Rodríguez, 2009). La respuesta metabólica en pacientes en estado crítico está caracterizada por notables incrementos en el gasto energético, catabolismo acelerado y cambios circulatorios hiperdinámicos (Chávez y Sánchez, 2009). Si estos cambios persisten, esta respuesta puede conducir a una pérdida considerable de masa corporal, disfunción orgánica y disminución de las funciones corporales reparativas e inmunes (Torres et al., 2008; Rodríguez, 2012). Tales procesos, especialmente si son exagerados, pueden producir malnutrición calórico-proteica, mayor morbilidad infecciosa, prolongada dependencia del ventilador, mayor estancia hospitalaria e incremento en la mortalidad (Kompan, Kremzar y Gardzijev, 1999; Omura, 2000).

Estos hallazgos han ocasionado un cambio en la orientación de la terapia nutricional: de simplemente ser un soporte en un estado hipermetabólico y alcanzar los requerimientos nutricionales a tener la oportunidad de manipular la respuesta inflamatoria e inmune local y sistémica (Seron, Zamora, Labarta y Mallor, 2013; Hernández y Chávez, 2008).

\section{(c) $\rightarrow$ (i)}


Las implicaciones de la terapia nutricional no son exclusivamente dietéticas, sino también un medio farmacológico a través del cual se mejora la función y estructura intestinal (con lo que se limita la progresión de la falla orgánica múltiple), un medio para mejorar la respuesta inmune y modificar la respuesta inflamatoria (Helmy, Vizcaychipi y Gupta 2007; Wang et al., 2013).

Dos terceras partes de todos los pacientes experimentan deterioro de su estado nutricional durante su estancia hospitalaria (Ninska de la Hoz, Camargo y Vargas, 2013). La enfermedad aguda exacerba el pobre estado nutricional del paciente al aumentar las demandas metabólicas y dificultar la utilización de los sustratos nutricionales. En nuestro caso, los pacientes en estado crítico frecuentemente reciben un inadecuado soporte nutricional durante su estancia en la unidad de cuidados intensivos (UCI), debido a que los médicos subestiman sus necesidades nutricionales, aunado a que las dietas enterales con inmunonutrientes no se contemplan dentro del cuadro básico de medicamentos de las instituciones de salud, lo que implica un gasto para el paciente; motivos por los cuales el inicio del apoyo nutricio a menudo se retrasa. Además, no se lleva un registro de los beneficios de la nutrición enteral temprana con inmunonutrientes (NETI), lo que hace que el médico no lo considere necesario (Fernández, Lobo, Ruiz y Pérez de la Cruz, 2009; Sánchez, Gutiérrez y Arzola, 2006).

La respuesta a la agresión puede manifestarse con una intensidad variable, dependiendo del grado de lesión. Sin embargo, el síndrome de desnutrición se presentará si no se cuenta con un ingreso nutrimental adecuado y oportuno. Al presentarse la desnutrición se puede comprometer la supervivencia y una evolución satisfactoria del paciente, lo que aumenta el riesgo de complicaciones infecciosas, días de estancia hospitalaria e incluso la disfunción orgánica múltiple y, por tanto, ocasionar la muerte (Vizzini y Aranda, 2011; Martinuzzi y Ferraresi, 2011; Jacobs et al., 2004).

Diversos estudios sugieren que el inicio del soporte nutricional en los pacientes dentro de las primeras 24 horas una vez ingresados a la UCI está asociado con una mejoría en el desenlace clínico, menores porcentajes de infección y una disminución del tiempo de hospitalización (Fuchs, 2009; Agudelo y Giraldo, 2008). La nutrición enteral se prefiere 
frente a la parenteral debido a que es más fisiológica, se asocia menos con la disfunción hepatobiliar y trastornos metabólicos y es mucho más económica (Ramírez, Gutiérrez, Domínguez y Barba, 2008).

La nutrición enteral se basa en el aporte de nutrientes con formulación químicamente definida por vía digestiva, utilizando el tracto gastrointestinal viable para su correcta absorción. Es un arma terapéutica para el médico, además de disminuir la translocación bacteriana y permitir un adecuado funcionamiento del sistema inmune intestinal (Rodríguez et al., 2012).

Por otra parte, si bien es una técnica que ha sido utilizada desde tiempos inmemoriales, en los últimos 25 años se ha experimentado un desarrollo considerable: pasó de ser un elemento terapéutico secundario, destinado exclusivamente a alimentar al paciente, a ocupar en la actualidad un papel importante que va mucho más allá del simple acto de nutrir (González y García, 2013).

La nutrición enteral temprana, definida como aquella que se inicia durante las primeras 24-48 horas de la lesión o ingreso a la UCI, es un método de soporte nutricional del cual se han descrito efectos benéficos para el paciente, adicionales al aporte calórico y proteico, como son el mantenimiento del tropismo intestinal y su implicación en la prevención de translocación bacteriana, estimulación de la motilidad intestinal y mejoría en la resistencia de los tejidos en cicatrización (Guzmán, 2008; Alted, Bermejo y Chico, 2009; MacFie, 2000).

Es sabido que el tiempo de hospitalización posterior a la lesión está en relación directa con el momento en que se reinicia la alimentación. Estas consideraciones permiten advertir la trascendencia del inicio temprano de la alimentación enteral, ya que los enfermos desnutridos se ven expuestos a periodos de curación más largos, padecen más complicaciones y presentan índices elevados de morbimortalidad con un incremento de los costos (Montoya y Múnera, 2014; Abdel, Abdel, Sánchez y Gómez, 2005; Leibson et al., 2012).

\section{(c) $\rightarrow$ (i)}


Desde el punto de vista biológico, además de su rol en la digestión y la absorción de nutrientes, el tubo digestivo regula y procesa activamente los sustratos circulantes y desempeña importantes funciones endocrinas, metabólicas e inmunológicas, al tiempo que actúa como una barrera eficiente evitando el pase de microorganismos y de sus productos de degradación a la circulación.

A su vez, el tubo digestivo se mantiene en gran parte a través de los elementos que se encuentran en su luz. La alimentación enteral aporta la mitad de los nutrientes del intestino delgado y más de $80 \%$ de los del intestino grueso; es la presencia de alimentos el estímulo más importante para su tropismo. De no existir ingesta, el tubo digestivo depende de principios endógenos como la glutamina o los cuerpos cetónicos que rápidamente se agotan, por lo que es imperioso reinstalar la alimentación lo más tempranamente posible (García y Grau, 2005).

La inanición conduce al acortamiento del yeyuno e íleon con disminución de la altura de las vellosidades, la caída de la celularidad y el deterioro de las uniones intercelulares (responsables de la función de barrera). En cambio, el reinicio de la alimentación estimula la producción de hormonas enterotróficas (enteroglucagón y gastrina), potencia la renovación de enterocitos y contribuye a mantener la altura de las vellosidades (García y Grau, 2005).

La alimentación enteral contribuye también a mantener la función intestinal, potencia la absorción de aminoácidos esenciales y estimula la defecación, lo que reduce la presión de penetración de las endotoxinas y bacterias. Igualmente, la respuesta inmune (celular y humoral) está influenciada por el ingreso de nutrientes (Mesejo y Blasco, 2000).

Por otra parte, la inmunonutrición nació a principios de los 90 con la idea de ser una vía con la cual, además de administrar al paciente los requerimientos nutricionales, se pudiera mejorar su estado inmunológico con el aporte de sustratos nutricionales con capacidad inmunomoduladora y así minimizar la respuesta a la agresión quirúrgica o traumática (Silva et al., 2016). Los componentes de estas dietas han demostrado la modificación de la respuesta inmune: favorecen la replicación de los linfocitos, estimulan la producción de determinados tipos de citoquinas y eicosanoides y disminuyen la liberación 
de otros, lo que lleva a la respuesta inflamatoria a un equilibrio, todo lo cual reditúa en una recuperación más rápida del paciente y, como se ha observado, en una disminución de la morbimortalidad, así como provoca el decremento de los días de estancia hospitalaria y, por extensión, la reducción de los costos tanto para el paciente como para la institución (Silva et al., 2016).

Teniendo en cuento todo lo hasta aquí mencionado, el presente estudio tiene como objetivo determinar el impacto de la NETI en la morbimortalidad en pacientes con traumatismo craneoencefálico (TCE) en la Unidad de Cuidados Intensivos del Hospital General de Mexicali, México, con el propósito de que, a partir de los resultados, se brinde información actualizada y relevante para el beneficio en el manejo nutricional del paciente.

\section{Material y métodos}

Se trató de un estudio clínico aleatorizado para determinar si la NETI en pacientes con TCE, en comparación con la nutrición enteral licuada (NETL), disminuye la morbimortalidad en la Unidad de Cuidados Intensivos del Hospital General de Mexicali. Dicho estudio se realizó del 1 de agosto de 2016 al 30 de junio de 2017. La muestra fue probabilística, aleatorizada. Se incluyeron 22 pacientes de 30 que ingresaron a la UCI con TCE (ver figura 1); pacientes que en el periodo previamente establecido, con diagnóstico de TCE, cumplieron con todos los criterios de inclusión (mayores de 18 años de edad, sexo indistinto, Glasgow $\leq 8$ y consentimiento informado por parte del familiar más cercano). Se consideraron, por su parte, los siguientes criterios de exclusión: pacientes con inestabilidad hemodinámica grave; pacientes con cualquier patología gastrointestinal mecánica o funcional; pacientes con expectativa de vida inferior a 24 horas; pacientes con desnutrición grave con los siguientes parámetros: IMC $<16 \mathrm{~kg} / \mathrm{m}^{2}$, albúmina $<2 \mathrm{~g} / \mathrm{dL}$ y $<800$ linfocitos $/ \mathrm{mm}^{3}$; pacientes con obesidad mórbida (IMC > 40), embarazo, enfermedad pulmonar obstructiva crónica (EPOC) o síndrome de inmunodeficiencia adquirida (SIDA), y pacientes incluidos en otros estudios de investigación.

\section{(c) $9 \Leftrightarrow$}

Esta obra está bajo licencia internacional Creative commons Reconocimiento-Nocomercial- 
Figura 1. Diagrama de flujo de participantes en el estudio

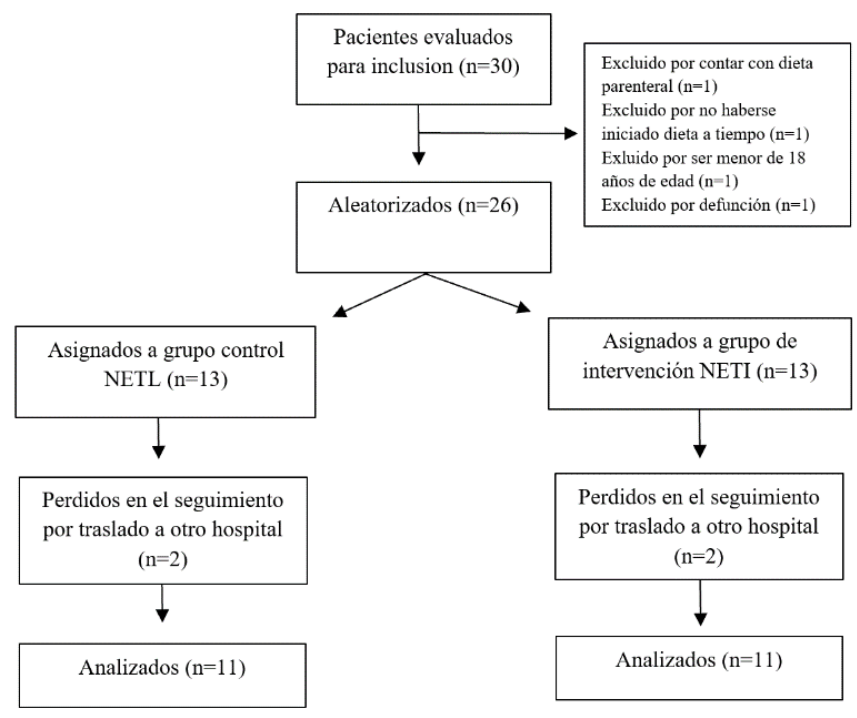

Fuente: Elaboración propia

La técnica de muestreo fue aleatorizada de la siguiente manera: el médico adscrito del área de cirugía (MAC) seleccionó y aleatorizó de manera sistemática a los pacientes según fueron ingresando a la UCI. Al grupo control se le administró NETL y al grupo intervención, NETI. En ambos casos dentro de las primeras 24 a 48 horas de estancia en la UCI. Además, el médico adscrito estableció un plan de manejo (protocolo de manejo en el paciente con TCE), medicación, exámenes de laboratorio y gabinete, medidas generales y asignó al paciente con TCE al grupo correspondiente (grupo NETL o NETI), así como realizó el llenado de la ficha del paciente (datos generales, valoración nutricional, antropometría, etc.). Posteriormente se realizó el cálculo de requerimientos energéticos y notificó al Departamento de Nutrición y Enfermería para la administración de la dieta según fuera el caso.

Previo al egreso de la UCI del paciente, se realizó de nuevo antropometría y se revisó que el expediente estuviera completo.

\section{(c) $\rightarrow \& \Leftrightarrow$}




\section{Selección de la fórmula enteral}

Para ambos grupos, se estimó $25 \mathrm{cal} / \mathrm{kg}$ al día, $33 \%$ de carbohidratos, $27 \%$ de proteínas y $40 \%$ de lípidos.

Al grupo control con NETL se le administró dieta polimérica en polvo (100 g): energía $330 \mathrm{kcal}$, carbohidratos 59.4 g, fibra 2 g, proteínas 17.5 g, lípidos 12.6 g, agua 1600 $\mathrm{ml}$, sodio $147 \mathrm{mg}$, potasio $790 \mathrm{mg}$. Se le agregó (licuó) al polvo: aceite de oliva $125 \mathrm{mg}$, fruta $80 \mathrm{~g}$, verdura $60 \mathrm{~g}$ y cereales sin grasa $19 \mathrm{~g}$, la cual fue elaborada por el Departamento de Nutrición del Hospital General y administrada por el personal de enfermería de la UCI. Se le administró la dieta al paciente intubado con la cabecera elevada a $30^{\circ}-45^{\circ}$, a través de sonda nasogástrica de 16 Fr colocada en bolsa de plástico para nutrición y su administración fue por gravedad durante 12 horas (50 cc/hora), dividida en dos tomas, por las mañanas y por las tardes. Se midió residuo gástrico, y se irrigó la sonda al término de cada sesión.

Por otra parte, al grupo de intervención con NETI se le administró la fórmula Supportan Drink, conformada de la siguiente manera: energía $300 \mathrm{kcal}$, kcal/ml 1.5, carbohidratos $24.80 \mathrm{~g}$ (33.0 \%), fibra $3 \mathrm{~g}$, proteínas $20 \mathrm{~g}(27.0 \%)$, nitrógeno $3.20 \mathrm{~g}$, lípidos $13.40 \mathrm{~g} \mathrm{(40.0 \% ),} \mathrm{agua} 76 \mathrm{ml}$, sodio $95 \mathrm{mg}$, potasio $256 \mathrm{mg}$, ácidos grasos omega-3 1429 mg, osmolaridad $435 \mathrm{mOsm} / \mathrm{L}$. La presentación de la botella era de $200 \mathrm{ml}$. Todo lo anterior más glutamine plus, que contiene $80 \mathrm{kcal}$ y 10 g de glutamina por sobre; se administró 30 g al día, la cual se elaboró y administró por el personal de enfermería de la UCI. De igual manera que en el otro grupo, se le administró la dieta al paciente intubado con la cabecera elevada a $30^{\circ}-45^{\circ}$, a través de sonda nasogástrica de 16 Fr colocada en bolsa de plástico para nutrición, aunque en este caso su administración fue por bomba de infusión durante 12 horas (50 cc/hora), dividida en dos tomas, por las mañanas y por las tardes. Se llevó a cabo una monitorización de la tolerancia de la nutrición enteral temprana de manera continua, vigilando la presencia de distensión abdominal, y la de canalización de gases, tipo de evacuaciones, presencia de volúmenes gástricos residuales $<500 \mathrm{ml}$ y evaluación de riesgo de aspiración.

\section{(c) 9 (9)}

Esta obra está bajo licencia internacional Creative Commons Reconocimiento-Nocomercial- 
Cabe mencionar que los investigadores, conscientes de que su interés primario es la salud de los enfermos, así como de la integridad de la investigación, no se encontraron influenciados por un interés secundario de tipo económico o personal, ya que ninguno de los miembros involucrados en la investigación percibió directa o indirectamente remuneración alguna por parte del fabricante de la fórmula.

\section{Resultados}

De los 22 pacientes incluidos en el estudio con TCE severo, $85 \%$ presentó una escala de coma de Glasgow a su ingreso de 3 (72.7 \% NETL y $100 \%$ NETI). Se incluyeron de manera aleatorizada 11 pacientes en el grupo A (grupo control) con NETL; y 11 en el grupo B (grupo de intervención) con NETI. En ambos grupos se inició la nutrición dentro de las primeras 24 a 48 horas de ingreso a la UCI. El cálculo de ambas dietas fue a razón de 25 $\mathrm{kcal} / \mathrm{kg} /$ día.

En relación con las características de la población, 16 fueron hombres (72.7\%) y 6 mujeres $(27.3 \%$ ), con una edad promedio de 34.7 años (en el grupo NETL, 5 pacientes en el rango de 18-32 años y 6 pacientes en el rango de 33-52 años; y en el grupo NETI, 6 pacientes en el rango de 18-32 años y 5 pacientes en el rango de 33-52 años). Las principales causas del TCE fueron por atropellamiento y accidente de tránsito (54.6\%), sin encontrarse diferencias significativas (tabla 1).

\section{(c) $9 \Leftrightarrow$}


Revista Iberoamericana de las Ciencias de la Salud

Tabla 1. Causas de TCE al ingreso a la UCI

\begin{tabular}{llll}
\hline Tipo de accidente & $\begin{array}{l}\text { NETL } \\
\text { Frecuencia (\%) }\end{array}$ & $\begin{array}{l}\text { NETI } \\
\text { Frecuencia }(\%)\end{array}$ & $\begin{array}{l}\text { Total } \\
\text { Frecuencia } \\
(\boldsymbol{\%})\end{array}$ \\
\hline Caída & 0 & $4(36.4)$ & $4(18.1)$ \\
Atropellamiento & $4(36.4)$ & $2(18.2)$ & $6(27.2)$ \\
Accidente de tránsito & $3(27.3)$ & $3(27.3)$ & $6(27.2)$ \\
Accidente de motocicleta & $1(9.1)$ & 0 & $1(4.5)$ \\
Agresión física & $3(27.3)$ & $2(18.2)$ & $5(22.7)$ \\
Total & $11(100)$ & $11(100)$ & $22(100)$ \\
\hline$(N=22)$ & & &
\end{tabular}

Fuente: Elaboración propia

Al evaluar el estado nutricional del paciente y su respuesta al trauma, al ingreso y egreso de la UCI, se encontró en el grupo con NETL una disminución de las mediciones antropométricas, disminución de albúmina y proteínas totales, de los linfocitos y leucocitos ( $\mathrm{t}$ de Student 3.308; $p=0.007$ ) (véase tabla 2).

Por otra parte, en el grupo con NETI se puede observar un aumento de peso ( $t$ de Student de 23.737; $p=0.001$ ), proteínas totales ( $\mathrm{t}$ de Student de 13.40; $p=0.001$ ), leucocitos (t Student de 2.05; $p=0.002$ ) y linfocitos ( $\mathrm{t}$ de Student de $7.09 ; p=0.001$ ) al egreso de la UCI (tabla 2).

\section{(C) $5 \Leftrightarrow$}


Revista Iberoamericana de las Ciencias de la Salud

Tabla 2. Características del estado nutricional en la población en estudio

\begin{tabular}{|c|c|c|c|c|}
\hline Características & $\begin{array}{l}\text { NETL } \\
\text { Media }(D S)\end{array}$ & & \multicolumn{2}{|c|}{$\begin{array}{l}\text { NETI } \\
\text { Media }(D S)\end{array}$} \\
\hline \multirow{2}{*}{ Total } & 11 & & 11 & \\
\hline & Ingreso & Egreso & Ingreso & Egreso \\
\hline $\operatorname{IMC}\left(\mathrm{kg} / \mathrm{m}^{2}\right)$ & $26.6(3.0)$ & $25.4(2.6)$ & $27.0(8.0)$ & $25.6(5.9)$ \\
\hline Peso (kg) & $54.1(18.2)$ & $52.4(17.9)$ & $\begin{array}{l}70.2 \\
(10.7)\end{array}$ & $\begin{array}{l}75.0 \\
(18.4) *\end{array}$ \\
\hline Pliegue tricipital $(\mathrm{cm})$ & $14.7(7.5)$ & $15.2(8.5)$ & $16.9(9.2)$ & $15.7(9.3)$ \\
\hline $\begin{array}{l}\text { Diámetro medio braquial } \\
(\mathrm{cm})\end{array}$ & $33.9(11.2)$ & $\begin{array}{l}33.4 \\
(11.4)\end{array}$ & $30.2(5.5)$ & $28.7(4.1)$ \\
\hline $\begin{array}{l}\text { Perímetro muscular } \\
\text { medio braquial }(\mathrm{cm})\end{array}$ & $37.2(24.7)$ & $17.2(9.2)$ & $27.2(7.1)$ & $26.5(5.6)$ \\
\hline Albúmina (g/dL) & $2.8(.86)$ & $2.3(.82)$ & $2.8(1.0)$ & $2.8(.63)$ \\
\hline Proteínas totales (g/dL) & $5.3(1.0)$ & $4.9(1.6)$ & $4.9(.96)$ & $5.5(1.3) *$ \\
\hline Leucocitos (mg/dL) & $9,793(37)$ & $\begin{array}{l}8,559 \\
(82.2) *\end{array}$ & $\begin{array}{l}19,732 \\
(17.0)\end{array}$ & $\begin{array}{l}25,343 \\
(40.8) *\end{array}$ \\
\hline Linfocitos (mg/dL) & $11.7(10.6)$ & $\begin{array}{l}11.17 \\
(10.6)\end{array}$ & $8.10(43)$ & $\begin{array}{l}15.4 \\
(7.2)^{*}\end{array}$ \\
\hline
\end{tabular}
$(\mathrm{N}=22) ; * \mathrm{p}<0.001$

Fuente: Elaboración propia

Los pacientes del grupo con NETI presentaron mayor tipo de patologías asociadas al TCE a su ingreso a la UCI, como hemorragia parenquimatosa, hematoma epidural, subdural y fracturas, sin encontrarse diferencias significativas (véase tabla 3).

\section{(C) $98 \mathrm{~S}$}


Revista Iberoamericana de las Ciencias de la Salud

Tabla 3. Patologías asociadas al TCE al ingreso a la UCI

$(N=22)$

NETL NETI

Morbilidad

$N=11$

$N=11$

Frecuencia (\%)

Frecuencia $(\%)$

Daño axonal difuso

$1(9.1)$

$1(9.1)$

Hemorragia

parenquimatosa

0

$1(9.1)$

Hematoma epidural

$1(9.1)$

$2(18.2)$

Hematoma subdural

$1(9.1)$

4 (36.4)

Fractura de cráneo

5 (45.5)

$2(18.2)$

Fractura costal

$2(18.2)$

3 (27.3)

Fractura de tórax

$1(9.1)$

$3(27.3)$

Otras facturas (pelvis, fémur)

0

\section{Fuente: Elaboración propia}

La principal morbilidad que se presentó en ambos grupos fue neumonía (diagnosticada por cuadro clínico, método radiológico y cultivo de secreciones bronquiales), y se encontró una significancia estadística en el grupo de NETI con neumonía (t de Student de $13.78, p=0.001$ ) e infección de vías urinarias diagnosticadas por general de orina y urocultivo ( $\mathrm{t}$ de Student de 8.07, $p=0.001$ ), en comparación con el grupo NETL (tabla 4).

\section{(c) $\rightarrow \& \Leftrightarrow$}


Revista Iberoamericana de las Ciencias de la Salud

Tabla 4. Morbimortalidad de la población en estudio

\begin{tabular}{lll}
\hline Morbimortalidad & NETL & NETI \\
& $\begin{array}{l}\boldsymbol{N}=\mathbf{1 1} \\
\text { Frecuencia }(\%)\end{array}$ & $\begin{array}{l}\boldsymbol{N}=\mathbf{1 1} \\
\text { Frecuencia }(\%)\end{array}$ \\
\hline Fiebre & $7(63.3)$ & $4(36.3)$ \\
Neumonía & $7(63.3)$ & $4(36.3) *$ \\
Infección de vías urinarias & $3(33.0)$ & $2(22.0) *$ \\
Falla renal & $2(18.2)$ & 0 \\
Choque séptico & $1(11.1)$ & 0 \\
Mortalidad & $1(11.1)$ & 0 \\
\hline
\end{tabular}

$N=22 ; * p<0.001$

Fuente: datos obtenidos del expediente clínico

Tanto los días de estancia en la UCI $\left(\mathrm{X}^{2}\right.$ Spearman -.432, $\left.p=0.05\right)$, de ventilación mecánica $\left(\mathrm{X}^{2}\right.$ Spearman -.432, $\left.p=0.05\right)$, como de nutrición enteral $\left(\mathrm{X}^{2}\right.$ Spearman -.425, $p=$ 0.05), fueron menores en el grupo de NETI en comparación con el de NETL (tabla 5).

Tabla 5. Estancia hospitalaria de la población en estudio

\begin{tabular}{|c|c|c|}
\hline $\begin{array}{l}\text { Características de la estancia } \\
\text { hospitalaria }\end{array}$ & $\begin{array}{l}\text { NETL } \\
N=11 \\
\text { Media }(D S)\end{array}$ & $\begin{array}{l}\text { NETI } \\
N=11 \\
\text { Media }(D S)\end{array}$ \\
\hline Días de ventilación mecánica en UCI & $16.1(10.2)$ & $9.7(6.0) *$ \\
\hline Días de estancia hospitalaria en UCI & $17.18(10.2)$ & $10.7(6.8) *$ \\
\hline Días de nutrición enteral en UCI & $15.9(10.3)$ & $9.7(6.0) *$ \\
\hline
\end{tabular}

Fuente: Elaboración propia

\section{(ब) 0 (9)}




\section{Discusión}

El presente estudio concuerda con estudios previos que han reportado que el TCE se presenta con más frecuencia en hombres que en mujeres, en una proporción 3:1, y que afecta principalmente a la población de 15 a 45 años.

Según se ha citado en estudios previos, las causas más frecuentes del TCE son accidentes de tráfico (42\%), caídas (23\%) y agresiones (14\%) (4); resultados similares se encontraron en el presente estudio, pues se reporta que la principal causa del TCE fue por accidente de tránsito $(27.20 \%)$ y atropellamiento $(27.20 \%)$, seguido por agresión física $(22.7 \%)$.

Como se mencionó anteriormente, el TCE suele presentarse en pacientes previamente sanos y con buen estado nutricional. A pesar de ello, los cambios metabólicos originados por la agresión traumática colocan a estos pacientes inmediatamente en una situación de riesgo nutricional (Ninska de la Hoz et al., 2013). Con referencia a lo anterior, se puede observar que $100 \%$ de los pacientes estudiados se encontraron a su ingreso a la UCI normonutridos en relación con las mediciones antropométricas; sin embargo, los niveles de albúmina, proteínas totales y leucocitos demuestran alteraciones propias del estado hipermetabólico. Al momento de su egreso, se observó una disminución en las mediciones antropométricas en ambos grupos, aunque en el grupo con NETI hubo un aumento de peso, la albúmina se mantuvo y se detectó un aumento de las proteínas, totales lo cual no se presenta en el grupo de NETL, cuyos integrantes entraron a una categoría de malnutrición moderada (Jiménez, 2003).

En estudios previos se reporta que los indicadores antropométricos no muestran diferencias significativas de desnutrición proteico-calórica. Sin embargo, se ha encontrado una tendencia a disminuir el peso corporal al egreso en $92.3 \%$ de los pacientes en relación con el ingreso (96.1\%) (Jiménez, 2003). Por otra parte, en estudios similares se ha señalado que estos indicadores antropométricos no son índices de gran especificidad para definir desnutrición proteico-calórica, ya que sus resultados llevan implícito cierto grado de error y median los cambios de los fluidos corporales (Ponce, Cornejo, Pérez y Mayagoitia, 2015)

\section{(C)}

Esta obra está bajo licencia internacional Creative commons Reconocimiento-Nocomercial- 
Diversos autores consideran que la albúmina sérica permite identificar a los pacientes con mayor riesgo de morbilidad y mortalidad, pero, debido a su vida media prolongada de 14 a 20 días, no refleja los cambios nutricionales rápidos; aun así, se toma este parámetro como referencia de desnutrición proteico-calórica (Arias, s. f.). Por otro lado, la albúmina sérica se ve afectada en situaciones de estrés o enfermedad aguda. De esta manera, en el paciente en estado crítico se ha encontrado que una concentración menor de $3.5 \mathrm{~g} / \mathrm{dL}$ se relaciona con un incremento de la morbilidad y mortalidad (Arias, s. f.). En el estudio de Jiménez (2003) se reporta hipoalbuminemia leve al egreso de la UCI, lo que coincide con los resultados obtenidos en el presente estudio.

Por otra parte, la determinación de proteínas totales es otro parámetro a considerar, y es un dato indirecto para evaluar el estado de las proteínas viscerales. En el presente estudio se encontró un aumento en los niveles séricos de proteínas totales al egreso de la UCI en el grupo con NETI; no así en el grupo con NETL.

Otro aspecto importante es la respuesta inmunoinflamatoria inicial del paciente con TCE, acompañada por un efecto inmunosupresor, debido a un exceso en la producción de citocinas, eicosanoides y al daño exudativo tisular, con un aumento de proteasas y radicales libres de oxígeno, provenientes de leucocitos polimorfonucleares y de macrófagos (Kieft et $a l ., 2005)$. Es por ello por lo que el recuento de leucocitos puede ser un marcador de la respuesta inmunológica del paciente. En estudios previos, $27.8 \%$ de los pacientes ingresados a la UCI presentaron disminución del recuento total de linfocitos (RTL) en los análisis hematológicos (Kieft et al., 2005), tal y como en el estudio de Jiménez (2003), donde 38.8 $\%$ de los pacientes desnutridos mostraron una disminución del RTL, y $23.6 \%$ de los pacientes no desnutridos también tenían afectado el RTL $(p<0.05)$. Resultados similares se encontraron aquí, donde se puede observar que el grupo con NETL presentó una disminución de leucocitos totales y linfocitos; no así en el grupo con NETI $(p<0.001)$.

El riesgo de adquirir infecciones nosocomiales es especialmente alto entre los pacientes de la UCI. Dicho riesgo de infección es 10 veces superior comparado con el resto de los pacientes ingresados en otras áreas del hospital. Según estudios europeos, en la UCI los principales tipos de infección son los siguientes: neumonía e infecciones respiratorias

\section{(c) $\rightarrow \& \Leftrightarrow$}

Esta obra está bajo licencia internacional Creative commons

Reconocimiento-Nocomercial- 
bajas (65\%), infección del tracto urinario (18\%) e infección del torrente sanguíneo (12\%). Un estudio americano similar encontró principalmente cuatro sistemas involucrados: 1) el tracto respiratorio $(31 \%), 2)$ el tracto urinario $(24 \%), 3)$ el torrente sanguíneo (16\%) y 4) las heridas quirúrgicas $(8 \%$.) $(17,18)$. En referencia a lo antes citado, y en relación con el presente estudio, se puede observar, al comparar ambos grupos (NETL vs NETI), la presencia de infecciones de vías respiratorias: $45.5 \%$ versus $36.4 \%$; y del tracto urinario: $36.4 \%$ versus $9.1 \%$. Esto es, se encontró una incidencia menor en los pacientes con NETI, lo cual concuerda con diversos estudios realizados en pacientes con TCE (Ramírez et al., 2008; Rodríguez et al., 2012; González y García, 2013).

Es por ello por lo que, actualmente, el criterio de nutrición en los pacientes críticos se ha modificado, con el objetivo fundamental de disminuir las complicaciones, como la infección y la reducción de la respuesta inflamatoria sistémica, independientemente de su estado nutricional al ingreso.

Por otra parte, en cuanto a la mortalidad, solo se presentó una defunción en el grupo con NETL, lo cual puede estar condicionado a la alimentación temprana administrada en ambos grupos, lo que demuestra que puede asociarse con menos infecciones y una tendencia hacia mejores resultados en términos de supervivencia (Ramírez et al., 2008; Rodríguez et al., 2012).

En estudios previos se reporta el efecto benéfico en cuanto a la disminución en la morbimortalidad y días de estancia hospitalaria en pacientes que han recibido NETI (Minard, Kudsk, Melton, Patton y Tolley, 2000). Hoy se conoce que todo paciente con trauma presenta un estado de inmunosupresión y por lo tanto un riesgo de sufrir infecciones nosocomiales.

En la actualidad, se ha comprobado que el uso de algunos inmunonutrientes en la dieta como la glutamina, arginina, ácidos grasos omega-3 y nucleótidos ocasionan una mejoría en la respuesta inmune en pacientes críticos, principalmente en el paciente con trauma. Los posibles efectos benéficos de la dieta con inmunonutrientes son varios y se encuentran descritos en la literatura, en particular la glutamina, ya que esta actúa como sustrato energético de células de división rápida (enterocito), de linfocitos y macrófagos (Rugeles, 2009).

\section{(C) GS}

Esta obra está bajo licencia internacional Creative Commons Reconocimiento-Nocomercial- 
Por otra parte, en el estudio realizado por Falcao de Arruda (2004) se reporta una disminución significativa de infecciones en pacientes con TCE al recibir dieta enriquecida con glutamina y probióticos. De esta manera, se hace hincapié en el efecto benéfico de la NETI para la prevención de infecciones en pacientes con TCE ingresados a la UCI, así como para la reducción de días de apoyo ventilatorio y de estancia hospitalaria.

En concordancia con lo anterior, en el presente estudio, a pesar de que el grupo con NETI presentó mayor tipo de patologías asociadas al TCE a su ingreso a la UCI, este cursó con menos días de apoyo ventilatorio mecánico, días de nutrición enteral y días de estancia hospitalaria. Al respecto, resultados similares se reportan en estudios previos en donde se puede apreciar que la nutrición adicionada con inmunonutrientes llevó a un menor número de días de estancia hospitalaria y de ventilación mecánica (González y García, 2013).

Así, pues, su empleo debe de formar parte de una estrategia terapéutica que minimice las complicaciones infecciosas, mantenga la efectividad nutricional y disminuya los días de estancia hospitalaria.

\section{Conclusiones}

Según los resultados obtenidos en el presente estudio, el uso de inmunonutrientes impacta de manera positiva en el paciente con traumatismo craneoencefálico, pues disminuye la incidencia de complicaciones infecciosas, así como el tiempo de estancia intrahospitalaria en la UCI. Y a partir lo anterior es posible mejorar en menos tiempo la salud del paciente y a la vez reducir costos debidos a hospitalización.

El mostrar los resultados obtenidos con la NETI al personal médico adscrito a la Unidad de Cuidados Intensivos como a la comunidad del Hospital General de Mexicali es muy importante, ya que se podrán romper paradigmas en cuanto a los beneficios de la nutrición temprana y al uso de inmunonutrientes, así como contribuir a nuevas líneas de investigación.

\section{(c) $9 \Leftrightarrow$}




\section{Referencias}

Abdel, O., Abdel, A., Sánchez, J. y Gómez, A. (2005) Nutrición enteral con dieta inmunomoduladora perioperatoria. Nutrición hospitalaria, 22(6),403-408.

Agudelo, G. M. y Giraldo, N. A. (2008). Soporte nutricional en el paciente crítico: una puesta al día. Revista de nutrición humana, 10(2), 191-211.

Alted, E., Bermejo, S. y Chico, M. (2009). Actualizaciones en el manejo del traumatismo craneoencefálico grave. Revista Med Intensiva, 33(1), 16-30.

Arias, Ma. C. (s. f.). La desnutrición en el paciente hospitalizado. Principios básicos de aplicación de la nutrición artificial. Guías Clínicas de la sociedad gallega de medicina Interna. Recuperado de http://www.meiga.info/guias/NutricionArtificial.pdf.

Chávez, J. P. y Sánchez, L. D. (2009). Impacto del retraso del inicio del apoyo nutricio en la morbimortalidad en la Unidad de Terapia Intensiva. Revista de la Asociación Mexicana de Medicina Crítica y terapia intensiva, 23(2), 70-75.

Falcao de Arruda, I .S. and De Aguilar, J. E. (2004). Benefits of early enteral nutrition with glutamine and probiotics in brain injury patients. Clin Sci (Lond), 106(3), 287-292.

Fernández, M., Lobo, G., Ruiz, M. D. y Pérez, A. (2009). Desnutrición hospitalaria: relación con la estancia media y la tasa de reingresos prematuros. Revista Medicina Clínica, 132, 377-384.

Fuchs, V. (1999). Alimentación vía enteral. Importancia en la práctica clínica. Revista de gastroenterología, México, 64, 95-100.

García, A. y Rodríguez, J. (2009). Traumatismo craneoencefálico y manejo nutricional del paciente neurológico en estado crítico. Nutr Hosp Suplementos, 2(2), 106-113.

García, B. y Grau, T. (2005). La nutrición enteral precoz en el enfermo grave. Nutrición Hospitalaria, 20(2), 93-100.

González, M. L. y García, A. (2013). Traumatismo craneoencefálico. Revista Mexicana de Anestesiología, 36(1), 186-193.

\section{(c) $\rightarrow \& \Theta$}

Esta obra está bajo licencia internacional Creative commons Reconocimiento-Nocomercial- 
Guzmán F. (2008). Fisiopatología del trauma craneoencefálico. Revista Colombia Médica, 39(3), 78-84.

Helmy, A., Vizcaychipi, M. and Gupta, A. K. (2007). Traumatic brain injury: intensive care management. Br J Anaesth, 99(1), 32-42.

Hernández, W. and Chávez, E. (2008). Nutrición enteral precoz en el paciente con lesiones complejas. Rev Cub Aliment Nutr, 18(2), 265-276.

Jacobs, D. G., Jacobs, D. O., Kudsk, K. A., Moore, F. A., Oswanski, M. F., Poole, G. V., Sacks, S., Scherer L. R. and Sinclair, K. E. (2004). Nutritional support in trauma patients. J Trauma. 57, 660-679.

Jiménez, S. D. (2003). Indicadores de desnutrición proteico calórica. Rev Cub Med Int Emerg, 2(4), 29-34.

Kieft, H., Roos, A. N., Van Drumen, J. D. E., Bindels, A., Bindels, J. C. y Hofman, Z. (2005). Eficacia de la inmunonutrición en los pacientes críticos. Medicina intensiva, 29(8), 446-448.

Kompan, L., Kremzar, B. and Gardzijev, E. (1999). Effects of early enteral nutrition on intestinal permeability and development of multiple organ failure after multiple trauma. Intensive Care Med, 25(2), 157-61.

Leibson, C., Brown, A., Hall, K., Ranson, J., Mandreak, J., Osler, T. and Malec, J. (2012). Medical care costs associated with traumatic brain injury over the full spectrum of disease: A controlled population-based study. Journal of neurotrauma, 29(11), 20382049.

MacFie J. (2000). Enteral versus parenteral nutrition: the significance of bacterial translocation and gut-barrier function. Nutrition, 16(7-8), 606-611.

Martinuzzi, A. L. y Ferraresi, E. (2011). Inmunonutrición y trauma. Rev cubana Aliment Nutr, 21(1), 129-146.

Mesejo, A. y Blasco, M. (2000). Manual básico de Nutrición Clínica y Dietética. Valencia, España: Generalitat Valenciana, Conselleria de Sanitat. Recuperado de https://www.mxgo.net/e-

\section{(c) $9 \Leftrightarrow$}

Esta obra está bajo licencia internacional Creative Commons Reconocimiento-Nocomercial- 
Revista Iberoamericana

de las Ciencias de la Salud

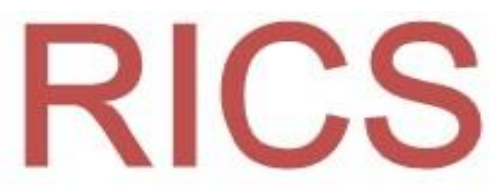

ISSN: 2395 - 8057

booksfree180511/8saludyestadofisico/Mesejo\%20Arizmendi\%20-

\%20Manual\%20Basico\%20De\%20Nutricion\%20Clinica\%20Y\%20Dietetica.pdf.

Minard, G., Kudsk, K. A., Melton, S., Patton, J. H. and Tolley, E. A. (2000). Early versus delayed feeding with an immune-enhancing diet in patients with severe head injuries. JPEN J Parenter Enteral Nutr, 24(3),145-149.

Montoya, S. and Múnera, N. (2014) Efecto de la intervención nutricional temprana en el resultado clínico de pacientes en riesgo nutricional. Nutrición hospitalaria, 29(2),427-436.

Ninska, A., Camargo, R. y Vargas, R. (2013). Caracterización del estado nutricional de pacientes adultos que ingresaron a una unidad de cuidado intensivo polivalente en la ciudad de Barranquilla. Acta Colombiana de Cuidado Intensivo, 13(3),153-168.

Omura, K., Hirano, K., Kanehira, E., Kaito, K., Tamura, M., Nishida, S., Kawakami, K. and Watanabe, Y. (2000) Small amount of low-residue diet with parenteral nutrition can prevent decreases in intestinal mucosal integrity, Am Surg, 231, 112-118.

Ponce, G., Cornejo, J. M., Pérez, M. E. y Mayagoitia, J. J. (2015). Nutrición enteral temprana en el paciente con traumatismo craneoencefálico: revisión sistemática de ensayos clínicos. Revista Iberoamericana de Ciencias de la Salud, 4(7), 1-17.

Ramírez, S., Gutiérrez, I. R., Domínguez, A. y Barba, C. (2008). Respuesta Metabólica al Trauma. Revista de Medicina Interna y Crítica, 5(4), 130-133. Recuperado de http://bvs.sld.cu/revistas/mie/vol2_4_03/mie06403.pdf.

Rodríguez, D., Rodríguez, C., Alfonso, L., Castellanos, E., Reyes, M. y Quintana, M. (2012). La respuesta metabólica en el trauma. Revista Cubana de Medicina Militar, 41(1),96-104.

Rodríguez, D., Rodríguez, C., Emerio, A. C., Castellanos, E., Reyes, M. y Quintana, M. (2012). Respuesta metabólica en el trauma. Revista Cubana de Medicina Militar, 41(1), 96-104.

Rugeles, S. J. (2009). Nutrición y metabolismo en Cirugía, nutrición y metabolismo en cirugía. Rev Colomb Cir, 24, 223-228.

\section{(c) $9 \Leftrightarrow$}

Esta obra está bajo licencia internacional Creative commons Reconocimiento-Nocomercial- 
Sánchez, V. M., Gutiérrez, J. J. y Arzola, C. (2006). Prealbúmina como marcador de seguimiento nutricional en pacientes críticamente enfermos. Revista de la Asociación Mexicana de Medicina Crítica y Terapia Intensiva, 20(1), 41-45.

Seron, C., Zamora, M., Labarta, L. and Mallor, T. (2013). Enteral nutrition in Critical Care. J Clin Med Res, 5(1), 1-11.

Silva, M. G., Asusu, N., Blumtritt, M., Kreff, Y., Lipovestky, F., Magnífico, L., Rebagliati, V., Vittal, A. y Yudi, M. (2016). Inmunonutrición en el paciente crítico. Diaeta, $34(155), 48-55$.

Torres, A., Fuentes, M., Jiménez, F., Sandoval, N., Vargas, B. y Ocampo, N. (2008). Nutrición enteral, intervención segura en la Unidad de Terapia Intensiva. Revista Medicina Crítica y Terapia Intensiva, 22(4), 226-235.

Vizzini, A. and Aranda, J. (2011). Nutritional support in head injury. Nutrition, 27(2), 129132.

Wang, X., Dong, Y., Han, X., Qi, X. Q, Huang, C. H. and Hou, L. H. (2013). Nutritional Support for Patients Sustaining Traumatic Brain Injury: A Systematic Review and Meta-Analysis of Prospective Studies. PLoS One, 8(3),1-14.

\section{(c) $98 \mathrm{~S}$}


Revista Iberoamericana de las Ciencias de la Salud

\begin{tabular}{|c|c|}
\hline Rol de Contribución & Autor (es) \\
\hline Conceptualización & $\begin{array}{l}\text { Gisela Ponce y Ponce de León, José Manuel Cornejo Bravo, María Eugenia Pérez } \\
\text { Morales y José de Jesús Mayagoitia Witrón (igual). }\end{array}$ \\
\hline Metodología & $\begin{array}{l}\text { Gisela Ponce y Ponce de León, José Manuel Cornejo Bravo, María Eugenia Pérez } \\
\text { Morales y José de Jesús Mayagoitia Witrón (igual). }\end{array}$ \\
\hline Software & $\begin{array}{l}\text { Gisela Ponce y Ponce de León (principal), Ulises Rieke Campoy y Alejandro } \\
\text { Mayagoitia Ponce (apoya). }\end{array}$ \\
\hline Validación & $\begin{array}{l}\text { Gisela Ponce y Ponce de León, José Manuel Cornejo Bravo, María Eugenia Pérez } \\
\text { Morales (igual). }\end{array}$ \\
\hline Análisis Formal & $\begin{array}{l}\text { Gisela Ponce y Ponce de León, José Manuel Cornejo Bravo, María Eugenia Pérez } \\
\text { Morales (igual) }\end{array}$ \\
\hline Investigación & $\begin{array}{l}\text { Gisela Ponce y Ponce de León, José de Jesús Mayagoitia Witrón, José Manuel } \\
\text { Cornejo Bravo, María Eugenia Pérez Morales (igual) }\end{array}$ \\
\hline Recursos & Gisela Ponce y Ponce de León, José de Jesús Mayagoitia Witrón (principal). \\
\hline Curación de datos & $\begin{array}{l}\text { Gisela Ponce y Ponce de León (principal), Ulises Rieke Campoy y Alejandro } \\
\text { Mayagoitia Ponce (apoya). }\end{array}$ \\
\hline $\begin{array}{l}\text { Escritura - Preparación del borrador } \\
\text { original }\end{array}$ & $\begin{array}{l}\text { Gisela Ponce y Ponce de León, Jose Manuel Cornejo Bravo y María Eugenia Pérez } \\
\text { Morales (igual). }\end{array}$ \\
\hline Escritura - Revisión y edición & $\begin{array}{l}\text { Gisela Ponce y Ponce de León, José Manuel Cornejo Bravo, María Eugenia Pérez } \\
\text { Morales y José de Jesús Mayagoitia Witrón (igual). }\end{array}$ \\
\hline Visualización & $\begin{array}{l}\text { Gisela Ponce y Ponce de León, José de Jesús Mayagoitia Witrón (igual), Ulises } \\
\text { Rieke Campoy y Alejandro Mayagoitia Ponce (apoya). }\end{array}$ \\
\hline Supervisión & Gisela Ponce y Ponce de León (principal). \\
\hline Administración de Proyectos & Gisela Ponce y Ponce de León y José de Jesús Mayagoitia Witrón (igual). \\
\hline Adquisición de fondos & Gisela Ponce y Ponce de León y José de Jesús Mayagoitia Witrón (igual). \\
\hline
\end{tabular}

\section{(c) $\rightarrow \mathrm{S} \theta$}

Esta obra está bajo licencia internacional Creative Commons Reconocimiento-NocomercialSinObrasDerivadas 4.0. 